\title{
Les mariages à la fātiha et le droit français
}

\section{Stéphane Papi}

\section{OpenEdition}

\section{Journals}

Édition électronique

URL : https://journals.openedition.org/rdr/648

DOI : $10.4000 /$ rdr.648

ISSN : 2534-7462

\section{Éditeur}

Presses universitaires de Strasbourg

\section{Édition imprimée}

Date de publication : 31 octobre 2017

Pagination : $129-140$

ISBN : 978-2-86820-974-0

ISSN : 2493-8637

\section{Référence électronique}

Stéphane Papi, «Les mariages à la fātiḩa et le droit français », Revue du droit des religions [En ligne], 4 2017, mis en ligne le 15 janvier 2020, consulté le 28 juin 2022. URL : http://journals.openedition.org/ rdr/648; DOl : https://doi.org/10.4000/rdr.648

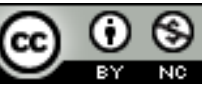

La revue du droit des religions est mise à disposition selon les termes de la Creative Commons Attribution - Pas d'Utilisation Commerciale 4.0 International - CC BY-NC 4.0. 


\section{LES MARIAGES À LA FĀTIḦA ET LE DROIT FRANÇAIS}

\section{Stéphane PAPI}

Docteur en droit, Chercheur associé, Aix-Marseille Université (IREMAM et LID2MS)

\section{RÉsumé}

Les mariages à la fätiha, constituent le fruit d'une synthèse entre les normes matrimoniales islamiques et les usages sociaux qui en sont faits chez les musulmans de France. Ils leur permettent de combiner des principes et des injonctions familiales avec des aspirations correspondant aux usages majoritairement en cours en France. Bien qu'ils puissent faire potentiellement l'objet de retraductions dans certaines de ses catégories juridiques, celles du concubinage ou des fiançailles, le droit français ne les reconnaît que s'ils sont célébrés postérieurement au mariage civil, ce dernier restant, chez une très large majorité de musulmans, l'acte qui scelle véritablement le mariage.

\section{Abstract}

Marriages in the fätiha amount to a synthesis between the Islamic matrimonial rules and the social uses that are made of them among the French Muslims. They allow them to combine family principles and injunctions, with aspirations corresponding to the predominant increasing usages in France. Although they might be considered as equivalent to well-known legal categories, like "concubinage" or engagement, French law recognizes marriages in the fätiha only if these are celebrated after the civil marriage, which remains, for a very large majority of Muslims, the act that actually seals marriage. 
T e mariage étant «[...] avec le feu et l'usage de l'outil, l'une des plus Lanciennes inventions de l'humanité ${ }^{1}$ », il constitue une institution de première importance se situant « au carrefour du droit et de la morale, de sorte que les pratiques matrimoniales tendent à se conformer à un modèle collectif qui reflète l'évolution sociale ${ }^{2} »$.

En France, ce modèle collectif a été marqué par la présence multiséculaire du catholicisme et bien qu'à la faveur d'un long mouvement historique de mutation des rapports entre les pouvoirs temporel et spirituel, le mariage soit devenu un acte uniquement civil, le droit français du mariage garde des traces de cette influence, tant au niveau des termes employés que des droits et devoirs respectifs auxquels sont soumis les époux ${ }^{3}$. Ceci illustre l'empreinte toujours présente des normes religieuses en droit français, car alors même que les autorités politiques et judiciaires ont à connaître du fait religieux ${ }^{4}$, nous verrons que la Cour de cassation s'y réfère parfois également.

L'émergence de l'islam, tout au moins dans l'Hexagone ${ }^{5}$, depuis la seconde moitié du siècle dernier est venue enrichir la palette des interactions entre le droit français et les normes religieuses. Celles-ci ont changé de nature, car les problématiques liées à la réception de normes issues de droits de pays arabo-musulmans, comme la polygamie et la répudiation, ou bien encore la kafäla (le recueil légal des mineurs) et ses relations avec l'adoption, s'effacent progressivement devant des problématiques de droit interne, les musulmans résidant désormais de manière définitive en France où beaucoup sont nés et en possèdent la nationalité. Alors qu'ils sont souvent présentés dans l'actualité politique et médiatique sous le seul prisme de l'antagonisme normatif, ce dernier ne suffit cependant pas à résumer les rapports actuellement à l'œuvre entre le droit français et les normes islamiques, qui sont tout à la fois issues

1. Meulders-Klein M.-Th., « L'évolution du mariage : de l'institution au contrat, et au-delà », in La personne, la famille et le droit. 1968-1998. Trois décennies de mutation en Occident, Bruxelles, Bruylant, Paris, LGDJ, 1999, p. 35.

2. Le Tertre C., La religion et le droit civil du mariage, Paris, Defrénois, 2004, p. 19.

3. L'article 165 du Code civil emploie le terme de « célébration» du mariage, ce qui constitue une claire référence au cérémonial chrétien. Le devoir de fidélité ou de secours (art. 212 du Code civil), l'obligation de monogamie (art. 147 du Code civil) sont des échos de prescriptions religieuses.

4. Cass. civ., 15 oct. 1991, cité in Madiot Y, « Le juge et la laïcité », Pouvoirs, n 75, 1995, p. 75.

5. Jusqu'en 1962, les normes islamiques ont été appliquées en Algérie française où les «indigènes » musulmans n'étaient majoritairement pas soumis aux dispositions du Code civil, mais relevaient d'un statut personnel et successoral de droit musulman ; jusqu'à très récemment, la polygamie était applicable aux habitants de l'île française de Mayotte, régis par un statut personnel local de droit musulman. 
de la sharī’a, la Loi divine au sens large - dont le fiqh, traduit par « droit musulman » ou «droit islamique » constitue la science de la compréhension - mais aussi d'un certain nombre de pratiques qui conservent auprès de la population musulmane un fort caractère normatif.

Ces rapports oscillent entre différentes variantes dont les classifications ne sont pas étanches mais évolutives, voire diverses ${ }^{6}$. Sans éluder les oppositions qui les caractérisent parfois, notamment celles issues du port du voile islamique sous ses différentes formes, il arrive que les normes islamiques soient intégrées sans difficulté en droit français, c'est le cas de la finance islamique, qu'elles fassent l'objet de compromis réciproques, comme en matière funéraire ${ }^{7}$, ou qu'elles donnent lieu à des retraductions dans le «langage programmatique » du droit français.

Les mariages religieux à la fätiḥa, ainsi dénommés car leur célébration donne lieu à la prononciation de la fätih̆a, c'est-à-dire la première sourate du Coran, relevant tout autant d'un usage social que d'une norme rattachée à l'islam se trouvent confrontés de différentes manières au droit français et peuvent faire potentiellement l'objet de retraductions.

\section{LE MARIAGE À LA FĀTIḦA OU LES USAGES SOCIAUX D'UNE NORME ISLAMIQUE EN FRANCE}

Les mariages à la fātiḥa, tels qu'ils sont pratiqués en France constituent le fruit d'une synthèse entre les normes matrimoniales islamiques et les usages sociaux qui en sont faits chez les musulmans de France.

\subsection{LES NORMES MATRIMONIALES ISLAMIQUES}

En islam, le mariage constitue un contrat synallagmatique ${ }^{8}$ « [...] placé par les auteurs dans la catégorie des contrats à prestations réciproques [...] par

6. PAPI S., « Normes islamiques et droit interne en France : de quelques zones de confluences », Droit et société, $\mathrm{n}^{\circ} 88,2014$, p. 689-708; « La Halal attitude et l'ordre juridique français», in Bergeaud-Blackler F., Les sens du halal. Une norme dans un marché mondial, Paris, CNRS Éd., 2015, p. 199-213.

7. PAPI S., « Droit funéraire et islam en France : l'acceptation de compromis réciproques », AJDA 2007, p. 1968-1973.

8. Le contrat est dit «synallagmatique » ou «bilatéral », lorsque ses dispositions mettent à la charge de chacune des parties ayant des intérêts opposés l'exécution de prestations qu'elles se doivent réciproquement. 
lequel un homme s'engage à verser une dot à une femme et à pourvoir à son entretien, en contrepartie d'avoir avec elle, licitement, des rapports intimes ${ }^{9} »$.

Ce contrat, parfois considéré comme " un acte purement civil sans coloration religieuse ${ }^{10} »$, n'en comporte pas moins une dimension religieuse essentielle. Il s'agit en effet d'une sunna (tradition) recommandée ${ }^{11}$. Le terme « mariage » (zawāğ $)$ figure plus de quatre-vingts fois dans le Coran ${ }^{12}$ qui en fait une "alliance sacrée et solennelle ${ }^{13} »$. La lecture de certains recueils de 'ahâdìt (dires) nous révèle plusieurs injonctions du Prophète Muhammad à se marier ${ }^{14}$, afin de préserver «[...] la moitié de sa religion ${ }^{15} »$.

Pour être valide, le mariage musulman ne doit pas comporter d'empêchements, soit permanents (notamment la parenté par le sang et l'allaitement qui lui est assimilé), soit temporaires (notamment d'ordre religieux - comme l'apostasie du conjoint ou la non-appartenance à l'islam du futur époux - ou d'ordre social, comme l'existence d'un nombre d'épouses supérieur à quatre). Le mariage doit également prévoir le versement d'une dot et comporter un échange de consentements.

La qualité de la personne qui exprime ce consentement diffère en fonction de celle des futurs époux. Lorsqu'ils sont impubères ${ }^{16}$, ils restent soumis au droit de contrainte matrimoniale (ğabr) exercé principalement par le père. Lorsqu'ils ont atteint l'âge de la puberté, la majorité des jurisconsultes malékites prévoient que la femme pubère peut consentir directement à son mariage en recourant aux services d'un walìy c'est-à-dire un tuteur matrimonial, ceux affiliés au rite hanéfite permettant à la femme pubère de consentir directement à son mariage, sans recourir aux services du walìy. C'est également le cas

9. Linant de Bellefonds Y., Traité de droit musulman comparé, Paris/La Haye, Mouton, 1965, p. 23.

10. Meziou K., JCl. Droit comparé. Tunisie. Droit civil - Droit de la famille, 2017, nº 82.

11. Naji El Mekkaoui R., La moudawanah : le référentiel et le conventionnel en harmonie. t. 1, Le mariage et la filiation, Rabat, Bouregreg, 2009, p. 78.

12. URvoy M.-Th., «La morale conjugale dans l'islam », Revue d'éthique et de théologie morale, $\mathrm{n}^{\circ} 240,3 / 2006$, p. 10.

13. Sourate IV, verset 21, trad. Blachere R., Le Coran, Paris, Maisonneuve et Larose, 1966, p. 107.

14. Muslim, Sahîh, nº 2485.

15. At-TABARÂNi et Al-HÂkim, $n^{\circ} 2681$.

16. Le garçon est soumis au droit de ğabr jusqu'à ce qu'il atteigne, selon les rites, 15 ou 18 ans. Selon le rite malékite, la fille, voire la femme y reste soumise jusqu'à la perte de sa virginité consécutive à la consommation d'un mariage légitime. Le rite hanéfite fait échapper la femme pubère à cette contrainte dès sa puberté, qu'elle soit vierge ou pas : Khillo I., Les droits de la femme à la frontière du droit international et du droit interne inspiré de l'islam: le cas des pays arabes, Aix-en-Provence, PUAM, 2009, p. 278. 
d'une femme qui a déjà été mariée (divorcée ou veuve) ${ }^{17}$. Le consentement des futurs époux doit relever de paroles explicites ne laissant aucun doute concernant la volonté des parties contractantes, les vices d'erreur ou de violence entraînant la nullité du mariage. Le caractère sacramentel des paroles prononcées à cette occasion, telle la prononciation de la fätih̆a ou d'autres versets coraniques n'est nullement exigé, tout comme la présence d'un imām, le mariage devant simplement être conclu en présence de deux témoins.

\subsection{LES USAGES SOCIAUX DU MARIAGE À LA FĀTIHA EN FRANCE}

On le voit, les mariages à la fätiha relèvent davantage d'un usage social que d'une norme islamique établie. Ils sont vécus par les intéressés et leurs familles comme un mariage halāl, c'est-à-dire un "bon mariage », religieusement licite, reconnu et valorisé par le groupe d'appartenance ${ }^{18}$, tout en relevant d'un syncrétisme religieux assez remarquable.

Alors même que dans les pays d'origine, ce sont les officiers d'état civil, voire les notaires ${ }^{19}$ traditionnels ('adūl) ${ }^{20}$ qui célèbrent et enregistrent les actes de mariage, en France, les mariages à la fātiḥa sont célébrés par les imāms, ou par des personnes reconnues pour leurs connaissances religieuses, au domicile des parents de l'un des époux, ou dans une mosquée, la prononciation de la fätiha étant assimilée à une condition de validité du mariage au point de le désigner. Les liens avec le mariage catholique sont saisissants : le prêtre, l'église et les prières...

Il arrive également que ces mariages soient qualifiés de fiançailles qui n'existent pas en tant que telles en islam et relèvent plutôt d'une tradition catholique tombée en désuétude.

Les mariages à la fätiḥa permettent aux futurs époux de respecter des principes et des injonctions familiales relevant davantage de pratiques traditionnelles que de normes islamiques, comme l'endogamie et le rôle traditionnellement prédominant dévolu aux parents et notamment aux mères dans la conclusion des alliances, ainsi que la virginité des futures épouses et le contrôle parental exercé sur leurs sorties hors de l'espace familial.

17. Muslim, Sahîh, nos 2544,2545 et 2546.

18. Collet B., SAntelli E., « Le mariage "halal", réinterprétation des rites du mariage musulman dans le contexte post migratoire français ", Recherches familiales, $n^{\circ}$ 9, 2012, p. 83.

19. Tunisie : Code du statut personnel art. 4 ; loi 1957, art. 31. Algérie : Code de la famille, art. 18 ; mais le mariage traditionnel qui n'est pas inscrit sur les registres d'état civil est rendu valide par jugement (Code de la famille, art. 22). Turquie : Code civil, art. 142.

20. Maroc : Code de la famille, art. 13 al. 4. 
Mais les mariages à la fätih̆a permettent également aux futurs époux de combiner ces principes avec des aspirations correspondant aux usages majoritairement en cours en France, à savoir le sentiment amoureux, fondateur du projet conjugal et son corollaire, le choix libre et assumé du futur conjoint, voire la possibilité d'avoir avec lui des relations intimes. Une fois la fätiḥa prononcée et l'union religieusement scellée, on pourra ainsi le fréquenter, dans ou en dehors de l'espace familial, selon que l'on se situe dans une famille plus ou moins traditionnelle ou, plus rarement, entamer une vie commune dans l'attente de la célébration du mariage civil. Les relations intimes apparaîtront alors licites, le péché de zinā' étant écarté, cette possibilité semblant être exploitée, a fortiori quand les mariages à la fätiḥa précèdent de plusieurs mois, voire de plusieurs années le mariage civil ${ }^{21}$.

L'adaptation au contexte français est également établie par la prééminence accordée chez la plupart des futurs époux et leurs familles au mariage civil constituant « [...] l'acte social qui entérine l'engagement pris devant le groupe d'appartenance ${ }^{22} »$. Il scelle donc véritablement le mariage, conformément au droit français.

\section{LE MARIAGE À LA FĀTIHA, DE LA CONFRONTATION À LA POSSIBLE RETRADUCTION EN DROIT FRANÇAIS}

Les mariages à la fätiha se trouvent confrontés de différentes manières au droit français et pourraient également faire l'objet de retraductions dans son « langage programmatique ».

\subsection{LES SITUATIONS DE CONFRONTATIONS ENTRE LE MARIAGE À LA FĀTIHY ET LE DROIT FRANÇAIS}

Les situations de confrontations avec le droit français sont d'une nature différente selon que l'on examine les problématiques liées au moment où est célébré le mariage à la fätiḥa et celles liées aux promesses des futurs époux concernant sa célébration.

21. Arslan L., "Union Halal : sexualité et mariage chez le couple "musulman" dans les quartiers populaires », in BeRGEAUd-BLACKLER F., op. cit., p. 141.

22. Collet B. et Santelli E., art. cit., p. 89. Seuls $8 \%$ des musulmans ne se marient que religieusement; SIMON P., TiBERJ V., Sécularisation ou regain religieux: la religiosité des immigrés et de leurs descendants, Paris, INED, 2013 (Documents de travail ; 196), p. 14. 
Après que le mariage civil a été instauré par la Constituante de 1791, il fut substitué au mariage religieux en septembre 1792, à la suite d'un long mouvement qui ne peut notamment pas être dissocié du fait que pendant longtemps, les mariages des protestants devant le pasteur et des juifs devant le rabbin ne pouvaient être enregistrés et étaient dénués de tout effet civil.

Aujourd'hui, en droit français, seul le mariage célébré par un officier d'état civil est officiel et pour bien marquer cette préséance, l'article 433-21 du Code pénal frappe d'une peine de 6 mois d'emprisonnement et $7500 €$ d'amende " tout ministre d'un culte qui procédera, de manière habituelle, aux cérémonies religieuses de mariage sans que ne lui ait été justifié l'acte de mariage préalablement reçu par les officiers de l'état civil ». L'article 433-22 du Code pénal prévoit également l'application de peines complémentaires comme l'interdiction des droits civiques, civils et de famille ainsi que l'interdiction, pour une durée maximale de dix ans, d'exercer une fonction publique ou d'exercer l'activité professionnelle ou sociale dans l'exercice ou à l'occasion de l'exercice de laquelle l'infraction a été commise.

Les époux, voire des tiers (parents ou amis) ainsi que d'autres ministres du culte pourraient être considérés comme complices de ce délit s'ils avaient incité, par encouragements, dons, promesses ou menaces le ministre du culte à célébrer ces mariages ou s'ils avaient aidé à leur préparation ${ }^{23}$.

Le Code pénal de 1994 a nettement adouci les sanctions, puisque d'instantanées - il suffisait que le ministre du culte ait célébré un seul mariage religieux avant le mariage civil - celles-ci sont devenues soumises à un délit d'habitude. Il faut donc que, désormais, soit établie contre le coupable la célébration d'au moins deux mariages religieux irréguliers, l'habitude étant constituée en droit pénal français dès le second acte défini par la loi ${ }^{24}$.

L'article 433-21 du Code pénal est une disposition de police et de sûreté au sens de l'article 3 du Code civil. La règle qu'il contient oblige par conséquent tous ceux qui résident sur le territoire, quelle que soit leur nationalité et sans avoir égard au contenu de leur statut personnel, donc même s'ils sont ressortissants de pays qui ne reconnaissent, comme civilement valable, que le mariage religieux, ou qui admettent la validité civile des mariages religieux à côté de celle des mariages célébrés devant un officier d'état

23. Vitu A., «Célébration d'un mariage religieux sans mariage civil préalable », JCl. Pénal, Art. 433-21, Fasc. 20, 2011, n 34.

24. Cass. crim., 24 mars 1944, DA 1944, p. 75, pour le délit d'avortement. - Legrain M., « Mariage civil et mariage religieux, se marier à l'église sans passer à la mairie », Rev. de droit canonique, 50-1, 2000, p. 163-169. 
$\operatorname{civil}^{25}$. La Cour de cassation a cependant jugé qu'un mariage religieux valablement conclu à l'étranger entre étrangers pouvait être reconnu en droit français ${ }^{26}$.

Alors qu'aujourd'hui cette règle de l'antériorité du mariage civil est acceptée par certaines organisations musulmanes ${ }^{27}$, les autorités semblent être informées du fait qu'elle n'est pas toujours respectée. Le ministère de l'Intérieur a ainsi reconnu que si la mosquée de Paris et les autres mosquées principales exigeaient la production d'un acte d'état civil préalable, ce n'était pas le cas de toutes les mosquées ${ }^{28}$. Le ministère de la Justice a également fait état des difficultés rencontrées dans la poursuite de cette infraction, la célébration des mariages à la fätiha préalablement au mariage civil bénéficiant de la complicité des mariés et des témoins et n'étant, de ce fait, pratiquement jamais dénoncée au procureur de la République ${ }^{29}$.

Dans une des rares affaires portées devant la justice, un imām poursuivi pour avoir régulièrement célébré des mariages religieux préalablement aux mariages civils arguait du fait que les prières de bénédiction récitées ne constituaient pas un mariage religieux, les fiancés s'étant mépris sur le sens de cette célébration. Les juges de la cour d'appel de Paris ont prononcé sa relaxe au motif que le sens de la cérémonie en cause n'était pas déterminé, le droit musulman ne conférant pas de fonction particulière à l'imām dans la célébration du mariage religieux, et qu'aucun registre des mariages religieux n'était tenu dans la mosquée où les cérémonies ont eu lieu ${ }^{30}$. Commentant cette décision, la doctrine a considéré que les juges ne devraient pas apprécier si les rites religieux accomplis étaient réellement ceux d'un mariage, mais seulement si le but recherché par les actes était de célébrer un mariage, quand bien même le rite n'aurait pas été respecté, cette position semblant la seule qui permette une application effective des dispositions de l'article 433-21 du Code pénal ${ }^{31}$.

25. CA Toulouse, 7 mai 1890, Journ. Parquets 1891, p. 211.

26. Cass. $1^{\text {re }}$ civ., 15 juin 1982, $\mathrm{n}^{\circ}$ 81-12.611, Bull. civ., I, $\mathrm{n}^{\circ} 224$.

27. Conseil européen de la fatwā : BoubeKeur A., « Le jeune marché matrimonial musulman en France : un espace entre spontanéité et institutionnalisation », Confluences Méditerranée, $\mathrm{n}^{\circ}$ 46, 2003, p. 166. - UNION DES ORGANISATIONS ISLAMIQUES DE FRANCE, Le mariage, entre acte civil et acte religieux, 23 oct. 2014 : http://www.uoif-online.com/islam-et-spiritualite/ mariage-acte-civil-acte-religieux [consulté le 26 juin 2017].

28. Rép. min. $\mathrm{n}^{\circ} 118221$, JOAN 28 mai 2007, p. 4319.

29. Rép. min. $\mathrm{n}^{\circ}$ 18053, JO Sénat $Q 25$ août 2011, p. 2225.

30. CA Paris, pôle 2, ch. 7,17 janv. 2013, n 11/08385, JurisData $\mathrm{n}^{\circ}$ 2013-001818.

31. MARÉCHAL J.-Y., « La difficile preuve du délit de célébration de mariages religieux avant le mariage civil », JCP G 2013, p. 427. 
C'était la position retenue par le tribunal correctionnel de Lorient en 1923, jugeant que s'il est indispensable, pour l'existence de l'infraction, que le ministre du culte ait procédé à une cérémonie religieuse, il importe peu que le mariage ainsi célébré soit religieusement valable. Les vices qui l'entacheraient ne font pas disparaître l'infraction et il suffit donc que ce mariage ait été regardé par le ministre célébrant lui-même comme valable ${ }^{32}$.

Les confrontations du droit français avec le mariage à la fätiha peuvent également s'opérer à son avantage, en ce sens que les juges peuvent annuler un mariage civil si un mariage religieux n'a pas été prononcé postérieurement à sa célébration. S'inscrivant dans un mouvement jurisprudentiel très ancien retenant le sentiment religieux comme une qualité essentielle de la personne du conjoint ${ }^{33}$, la cour d'appel d'Aix-en-Provence a ainsi considéré concernant deux époux musulmans, que le consentement au mariage s'entend par le consentement donné à la totalité des cérémonies, tant civiles que religieuses et que le refus d'un des époux de se soumettre au mariage religieux constitue, pour l'autre, une erreur sur les qualités substantielles justifiant l'annulation du mariage sur le fondement de l'article 180 du Code civil ${ }^{34}$.

\subsection{LES POSSIBLES RETRADUCTIONS DU MARIAGE À LA FĀTIHA DANS LE « LANGAGE PROGRAMMATIQUE » DU DROIT FRANÇAIS}

Ce phénomène de retraduction s'opère dans ce que Pierre Bosset et Paul Eïd ont appelé, en faisant référence à l'ouverture du droit étatique au pluralisme juridique, « le langage programmatique » du droit, cette opération ne provoquant pas l'introduction pure et simple d'une norme non étatique dans le système juridique, mais en facilitant sa reconnaissance symbolique ${ }^{35}$. Si les retraductions ci-après évoquées sont le fruit d'hypothèses théoriques, leur évocation permet de cerner les potentialités de développement qu'elles recèlent à la faveur de jurisprudences à venir.

Les mariages à la fātiḩa pourraient tout d'abord être retraduits dans la catégorie juridique du droit français correspondant aux fiançailles, ce terme

32. T. corr. Lorient, 29 oct. 1923, Gaz. Pal. 1924, 2, p. 68.

33. CA Colmar, 6 déc. 1811, S. 1811, 2, p. 588 : il s'agissait d'un homme qui, ayant caché sa qualité de frère laïc capucin, s'était marié ; la cour d'appel de Colmar annula le mariage.

34. CA Aix-en-Provence, 15 nov. 2005, JurisData n 2005-293158, Dr. Fam 2006, comm. 80.

35. Bosset P., EID P., « Droit et religion : de l'accommodement raisonnable à un dialogue inter normatif ?", Commission des droits de la personne et des droits de la jeunesse Québec, avril 2006, Cat. 2.500.127. 
étant d'ailleurs souvent employé par les futurs mariés et leurs familles pour désigner ce type d'union.

Les fiançailles sont considérées comme une situation de fait, la preuve peut en être rapportée par tous moyens ${ }^{36}$, notamment par l'existence d'une cérémonie religieuse consacrant la promesse de mariage ${ }^{37}$, ce cas de figure correspondant tout à fait aux mariages à la fätiḥa. Les fiançailles permettent d'invoquer un préjudice matériel et moral en cas de décès d'un fiancé et le versement de dommages et intérêts ${ }^{38}$. Le préjudice matériel résulte des frais exposés en vue du mariage ${ }^{39}$, alors que le préjudice moral est caractérisé lorsque, par exemple, la rupture cause déception et humiliation au fiancé délaissé ${ }^{40}$. La rupture des fiançailles peut justifier la restitution des cadeaux ${ }^{41}$. Concernant la bague de fiançailles, sa restitution dépend de la valeur symbolique de ce bijou ou de la situation économique du donateur, ces deux conditions pouvant être cumulatives ${ }^{42}$. Si le don entre fiancés porte sur des bijoux qui ont un caractère familial marqué et se transmettent par exemple de génération en génération, la restitution est obligatoire après la rupture ${ }^{43}$. La rupture des fiançailles peut aussi donner lieu au partage de certains biens qui auraient été acquis par les deux fiancés ${ }^{44}$.

Les mariages à la fātiḥa pourraient également être retraduits dans la catégorie juridique du droit français correspondant au concubinage. Ce cas de figure sera cependant beaucoup plus rare, car le concubinage, à la différence des fiançailles, suppose d'entamer une vie commune ${ }^{45}$, ce que ne font généralement pas les mariés à la fätiḥa ${ }^{46}$.

36. Cass. $1^{\text {re }}$ civ., 6 nov. 1974, n $^{\circ} 73-10.029$, Bull. civ., I, n 296, p. 254.

37. CA Bordeaux, 17 juin 1998, n 96005945 , JurisData ${ }^{\circ}$ 1998-045062.

38. CA Rouen, 9 juill.1952, D. 1953, Jur., p. 13. - CA Rennes, 30 juin 1983, D. 1986, IR, p. 64.

39. CA Rouen, 15 juin 2005, $n^{\circ}$ 03/01005, JurisData $n^{\circ}$ 2005-278689. - CA Aix-en-Provence, 3 mars 2005, $\mathrm{n}^{\circ}$ 03/07185, JurisData $\mathrm{n}^{\circ}$ 2005-271242. - CA Douai, 28 oct. 2002, $\mathrm{n}^{\circ}$ 01/04301, JurisData $\mathrm{n}^{\circ}$ 2002-209226.

40. CA Aix-en-Provence, 3 mars 2005, n 03/07185, JurisData n 2005-271242. - CA Montpellier, 7 déc. 2004, n 03/05372, JurisData ${ }^{\circ}$ 2004-266639. - CA Bordeaux, 17 juin $1998, n^{\circ} 96005945$, JurisData ${ }^{\circ} 1998-045062$.

41. CA Paris, 22 nov. 1949, D. 1950, Jur., p. 97. - Cass. $1^{\text {re }}$ civ., 19 déc. 1979, D. 1981, Jur., p. 449. - CA Versailles, 22 nov. 2002, AJ Fam. 2003, p. 286.

42. CA Paris, 13 oct. 2004, $n^{\circ}$ 03/19688, JurisData ${ }^{\circ}$ 2004-264379.

43. Cass. $1^{\text {re }}$ civ., 19 déc. 1979, D. 1981, Jur., p. 449.

44. Pour un prêt, V. CA Paris, 5 juin 1981, JurisData n 1981-025104.

45. Code civil, art. 515-8. Une vie commune qui succède à un mariage religieux constitue un concubinage : CA Paris, 8 déc. 1992, JurisData n 1992-023694.

46. Collet B. et Santelli E., art. cit., p. 89. 
En cas de rupture, le concubin ne devra pas laisser sans ressources celui ou celle « qui lui a consacré une partie de sa vie et apporté son soutien moral ou matériel ${ }^{47}$ », le partage des biens devant être opéré par moitié entre les concubins $^{48}$. En cas de décès, le concubin survivant a également le droit de réclamer réparation au responsable du décès accidentel de son compagnon ${ }^{49}$. Si le décès résulte d'un accident professionnel, la concubine bénéficie du même droit à indemnisation que la femme mariée ${ }^{50}$.

\section{CONCLUSION}

Préalablement à l'adoption de la loi du 17 mai 2013 ouvrant le mariage aux couples de personnes de même sexe, les représentants des différentes religions de France ont exprimé leur opposition quasi unanime à ce qui n'était encore qu'un projet de loi. Leurs critiques, pratiquement similaires, portaient sur la remise en cause du caractère institutionnel du mariage provoquant une contractualisation et une individualisation de la vie familiale jugée trop prononcée $^{51}$. Des voix assez fortes dans la sphère catholique traditionaliste ont considéré que cette loi rompait le consensus paisible existant entre l'État et l'Église catholique qui reconnaissait le mariage civil parce qu'il constituait une institution visant à assurer la stabilité à la filiation ${ }^{52}$.

En conséquence de quoi une revendication visant à supprimer l'obligation d'antériorité du mariage civil sur le mariage religieux a vu le jour, relayée sur la scène politique par plusieurs députés et sénateurs qui ont déposé des amendements en ce sens à l'occasion de l'examen par le Parlement du projet de loi, amendements qui ont tous été rejetés. Ceux-ci faisaient référence au

47. Cass. $1^{\text {re }}$ civ., 17 nov. 1999, n 97-17.541, JurisData ${ }^{\circ} 1999-004082$.

48. Cass. $1^{\text {re }}$ civ., 19 avr. 2005, $\mathrm{n}^{\circ}$ 01-17.226, JurisData $\mathrm{n}^{\circ} 2005-028145$.

49. Cass. crim., 10 nov. 1992, Bull. inf. C. cass. 1992, n 361.

50. Code de la Sécurité sociale, art. L. 434-8.

51. Michel J.-P., Rapport [...] sur le projet de loi adopté par l'Assemblée nationale ouvrant le mariage aux couples de personnes de même sexe, Paris, Sénat, 2013, n 437, t. 2, auditions du 12 févr. 2013, p. 58-83.

52. Pour le Père Cédric BuRgun, le mariage civil « [...] se conçoit aujourd'hui uniquement comme un acte individualiste et personnel s'enracinant dans la compréhension révolutionnaire des droits de l'homme. Le "mariage pour tous" ne sera qu'un acte de plus dans cette "individualisation" de l'homme et du mariage, coupé de la société et de son histoire » : «La question est de savoir si nous voulons donner un statut légal à l'homosexualité », 25 févr. 2013 : http://lesalonbeige.blogs.com; V. également l'appel de l'abbé Pillet pour préserver le mariage religieux, 12 sept. 2013 : http://www.hommenouveau.fr/663/religion/ appel-de-l-abbe-pillet-pour-preserver-le-mariage-religieux.htm [consultés le 26 juin 2017]. 
fait que les sanctions prévues par le Code pénal étaient contraires à l'article 9 de la Convention européenne des droits de l'homme et constituaient une restriction à la liberté de manifester sa religion ${ }^{53}$.

Si l'on ne peut pas prédire l'issue de recours déposés contre la loi française sur cette base, on constatera que la Cour européenne des droits de l'homme, dans un arrêt rendu en 2010 a décidé, à l'unanimité, que le refus des autorités turques de considérer une femme comme l'ayant droit d'un homme avec lequel elle n'avait contracté qu'un mariage religieux n'était pas contraire à la Convention, ce qui revient à admettre que le mariage religieux puisse ne pas être reconnu juridiquement ${ }^{54}$.

De plus, bien qu'il semble que certains musulmans appartenant aux courants rigoristes de l'islam refusent le mariage civil considéré comme "illégitime ${ }^{55}$ ", ces revendications ne sont pas soutenues par les organisations musulmanes de France et ne semblent pas correspondre aux attentes des musulmans ${ }^{56}$, dont nous avons vu qu'ils accordent d'ordinaire la prééminence au mariage civil.

De ce fait, et aussi car elle heurterait un principe fortement ancré en droit français, leur reconnaissance juridique est donc improbable, à moins que l'action des milieux catholiques traditionalistes ne la favorise indirectement.

53. V. les amendements déposés par le sénateur Hervé Portelli ainsi que ceux déposés par les députés M. de Courson, M. Le Fur, M. Breton, M. Decool et M. Sermier.

54. CEDH, Gde ch., 2 nov. 2010, n 3976/05, Şerife Yiğit c/ Turquie.

55. ArsLan L., précit., p. 143.

56. Même si, selon une enquête publiée au mois de septembre 2016 par l'Institut Montaigne, $28 \%$ des musulmans de France penseraient que la loi religieuse est plus importante que la loi de la République, cette opinion allant jusqu'à représenter plus d'un tiers des sondés chez les jeunes de moins de 30 ans : El Karour H., Un islam français est possible, Paris, Institut Montaigne, sept. 2016 : http://www.institutmontaigne.org/fr/publications/ un-islam-francais-est-possible\# [consulté le 26 juin 2017]. 